\title{
Fast AC Power Flow Optimization Using Difference of Convex Functions Programming
}

\section{Journal Article}

Author(s):

Merkli, Sandro; Domahidi, Alexander; Jerez, Juan L.; Morari, Manfred; Smith, Roy (D)

Publication date:

2018-01

Permanent link:

https://doi.org/10.3929/ethz-b-000318769

Rights / license:

In Copyright - Non-Commercial Use Permitted

Originally published in:

IEEE Transactions on Power Systems 33(1), https://doi.org/10.1109/TPWRS.2017.2688329 


\title{
Fast AC Power Flow Optimization using Difference of Convex Functions Programming
}

\author{
Sandro Merkli ${ }^{1,2}$, Alexander Domahidi ${ }^{2,3}$, Juan Jerez ${ }^{1,3}$, Manfred Morari ${ }^{1}$, Roy S. Smith ${ }^{1}$
}

\begin{abstract}
An effective means for analyzing the impact of novel operating schemes on power systems is time domain simulation, for example for investigating optimization-based curtailment of renewables to alleviate voltage violations. Traditionally, interior-point methods are used for solving the nonconvex AC optimal power flow (OPF) problems arising in this type of simulation. This paper presents an alternative algorithm that better suits the simulation framework, because it can more effectively be warm-started, has linear computational and memory complexity in the problem size per iteration and globally converges to Karush-Kuhn-Tucker (KKT) points with a linear rate if they exist. The algorithm exploits a differenceof-convex-functions reformulation of the OPF problem, which can be performed effectively. Numerical results are presented comparing the method to state-of-the-art OPF solver implementations in MATPOWER, leading to significant speedups compared to the latter for smaller systems and comparable performance for larger cases.
\end{abstract}

\section{INTRODUCTION}

The amount of renewable energy sources (RES) in distribution systems is steadily increasing [1]. Due to their volatility and limited predictability, they are posing new challenges to power system operation and planning. A prominently observed consequence of the increase in renewable power infeeds are local voltage limit violations [2]. Traditionally, the remedy for these violations required expensive line capacity extensions. Recent studies have shown that such extensions could be reduced by a shift in operational paradigms from rule-based to optimization-based approaches, see for example [3]-[5].

Since it is non-trivial to predict the impact of such shifts in operational paradigms on power systems, time-domain simulations provide valuable insight [4]. System-wide simulations over extended periods of time can demonstrate seasonal impacts and yield statistical data. This data provides a more in-depth view than worst-case snapshot studies, which are the current industrial practice. While the latter only provides information on violation severity, the former also gives a sense of how often they occur. However, if the impact of optimization-based approaches is to be simulated over such long periods of time and for different scenarios, a large number of optimization problems need to be solved. In the case of dispatch optimization, sampling times for the control

\footnotetext{
${ }^{1}$ Automatic Control Lab, ETH Zurich, Physikstrasse 3, 8092 Zurich \{merkli, smith, juanj, morari\}@control.ee.ethz.ch

${ }^{2}$ Inspire-IfA, Inspire AG, Technoparkstrasse 1, 8005 Zurich. \{merkli, domahidi\}@inspire.ethz.ch

${ }^{3}$ embotech GmbH, Physikstrasse 3, ETL K10.1, 8092 Zurich, \{jerez, domahidi\}@embotech.com
}

are on the order of 15 minutes. This means that proposed optimization problems can typically be solved fast enough for on-line operation using state-of-the-art software such as MATPOWER [6]. However, in simulations, solving the optimization problems is the most computationally expensive task. Therefore, efficient numerical methods are essential for performing simulations in a practical time frame.

In many cases, the problems proposed in optimizationbased operation schemes are related to a class of problems collectively referred to as optimal power flow (OPF) problems. An extensive amount of literature exists on solving such problems and a recent survey is given in [7], [8]. However, due to the non-convexity and large scale of the problem, it remains an active research topic. In fact, the nonconvexity makes the problem computationally intractable to solve to global optimality in general. However, critical points can in most cases be found efficiently if they exist. The most popular approaches to solving AC OPF problems are interior point methods [9] and sequential convex approximation methods [10]-[12]. While the former are numerically robust and well-studied, the latter tend to be faster according to [7]. There are two main sequential convex approximation approaches: Sequential linear programming (SLP) and SQP. These schemes approximate the original problem iteratively with convex linear and quadratic programs, respectively. Most implementations of these approaches use conventional power flow computations between their iterations to restore feasibility of the Kirchhoff equations. In general, SLP/SQP methods require extensions to become globally convergent, which reduces their performance [7]. A recently developed alternative approach is to solve a convex semidefinite programming (SDP) relaxation of the problem [13], [14]. The optimal value of this relaxation is either the globally optimal value of the non-convex problem or in the worst case only a lower bound on the latter.

The reformulation presented in this paper allows for the solution of AC optimal power flow problems using difference of convex functions programming [15]. In comparison with SLP, the method has no issues of unboundedness of the relaxations and is globally convergent without extensions. The presented method operates entirely in the voltage space, satisfying the Kirchhoff equations by design and thereby eliminating the need for conventional power flow computations. In comparison with the SDP relaxation, it converges to critical points at a lower computational cost than the former. Also, the SDP relaxation provides only a lower bound on the objective in the worst case, which does not provide a feasible 
point. Whether or not the SDP relaxation holds also depends on the set of active constraints, which can be different for different operating points of the same system. In contrast, the proposed method always converges to a critical point if one exists. While local optimality of these points is not guaranteed, experiments show that they represent acceptable solutions. This statement will be quantified in the numerical results section.

In this work, we present the application of our method to a specific example of an optimization-based operation scheme designed to reduce RES curtailments. In this example, the distribution system operator (DSO) is tasked with keeping the system stable and within the allowed operating conditions. Normally, DSOs are state-regulated entities, thus their operation is not for financial gain. One example of such a regulation is the EEG in Germany [16]. The range of actions the DSO can take includes adjusting setpoints of generators and curtailing renewable energy sources. Approaches for finding such points currently used in practice are usually rule-based. Such rules involve a significant amount of tuning and rarely come with mathematical guarantees. Additionally, costs for adjustments can only indirectly be taken into account. With an optimization-based approach, such costs can naturally be handled.

While the rest of the paper is developed with this specific example in mind, the theory applies to a wide range of problems involving similar constraints, including standard economic dispatch. In particular, any AC power flow optimizations can make use of the decomposition technique presented here.

\section{A. Outline}

The rest of this paper is structured as follows: Section II introduces some preliminaries. In Section III, we present an efficient reformulation of the optimization problem such that the difference-of-convex-functions method is applicable. Section IV elaborates on how the convex inner problems arising in the proposed algorithm can be solved efficiently. In Section V, numerical results are presented and discussed. Final conclusions are presented in Section VI.

\section{Preliminaries}

This section outlines both the model of the power system as well as the optimization-based control strategy we propose. Basic notation is introduced, assumptions are clarified and current operational practice is described.

\section{A. Notation}

The power grid is modeled as an undirected graph with $N$ vertices and $L$ edges. Let the edge and vertex sets be $\mathcal{E}$ and $\mathcal{N}$, respectively. Vertices model buses, while edges model power lines. Each line (say, from bus $j$ to bus $l$ ) has admittance $y_{j l} \in \mathbb{C}$. Each bus $j$ has an associated voltage $v_{j} \in \mathbb{C}$ and power in-feed $s_{j} \in \mathbb{C}$, where $\operatorname{Re}\left(s_{j}\right)$ denotes active and $\operatorname{Im}\left(s_{j}\right)$ denotes reactive power. Let $v, s \in \mathbb{C}^{N}$ be the stacked versions of the bus voltages and powers, respectively. The admittance matrix of the grid is given as

$$
Y_{j l}:= \begin{cases}y_{j l} & \text { if } j \neq l, \\ y_{j}^{\mathrm{sh}}-\sum_{k=1, k \neq j}^{N} y_{j k} & \text { if } j=l .\end{cases}
$$

where $y_{j}^{\text {sh }} \in \mathbb{C}$ are shunt admittances. The Kirchhoff equations for the system can hence be written in matrix form:

$$
\operatorname{diag}(v) \bar{Y} \bar{v}=s,
$$

where ${ }^{-}$describes the (element-wise) complex conjugate. Let $e_{k}$ denote the $k$-th unit vector with appropriate dimension. Let $(\cdot)^{r}:=\operatorname{Re}(\cdot),(\cdot)^{q}:=\operatorname{Im}(\cdot)$ and let $g_{k}, b_{k}$ be the $k$ th rows of $\operatorname{Re}(Y)$ and $\operatorname{Im}(Y)$, respectively. For symmetric matrices $A \in \mathbb{C}^{n \times n}$, let the set $J(A)$ be the set of indices of non-zero columns.

\section{B. Operational constraints}

The constraints representing limits introduced by the system operator are either due to regulations or to avoid damage to the system. Firstly, the voltage magnitude has to be within a fixed interval for each bus $j$ :

$$
v_{\min , j} \leq\left|v_{j}\right| \leq v_{\max , j}
$$

These limits are important for distribution grids, since the assumption of low-resistance lines commonly made in transmission grids does not hold. This means there can be significant discrepancies in the voltages between two endpoints of a line. Additionally, one of the main problems faced by DSOs are voltage constraint violations due to local renewable power in-feeds. Finally, the current through each line $(j, k)$ is limited for thermal reasons:

$$
\left|y_{j l}\right|\left|v_{j}-v_{l}\right| \leq i_{\max , j l}
$$

The limits in (3) and (4) together will hereafter be referred to as the operational constraints for the power grid. The DSO action space is modeled as an interval of active and reactive power for each bus $j$ :

$$
\begin{aligned}
p_{\min , j} & \leq \operatorname{Re}\left(s_{j}\right) \leq p_{\max , j} \\
q_{\min , j} & \leq \operatorname{Im}\left(s_{j}\right) \leq q_{\max , j} .
\end{aligned}
$$

For buses at which the DSO cannot intervene, the upper and lower limits in (5) are equal. Let $\left(s_{0}, v_{0}\right)$ be an operating point of the power grid that represents the state of the distribution grid without any optimization-based intervention. If this point satisfies all operational constraints (3) and (4), no DSO intervention is required. Otherwise, some limits are violated and the task of the DSO is then to find a point $(s, v)$ that satisfies all operational constraints, but also lies within its action space (5).

\section{DSO optimization problem}

The penalization for introduced deviations to power setpoints is modeled linearly here, while voltage deviations are interpreted as an effect of changing powers without a direct cost. This is the case for example in Germany [16]. Even though the DSO is not run for profit, its operational cost 
has to be covered by the power consumers. It is therefore reasonable to perform a social welfare optimization for least cost:

$$
\begin{array}{ll}
\underset{s \in \mathbb{C}^{N}, v \in \mathbb{C}^{N}}{\operatorname{minimize}} & \left\|\operatorname{Re}\left(s-s_{0}\right)\right\|_{1}+\left\|\operatorname{Im}\left(s-s_{0}\right)\right\|_{1} \\
\text { subject to } & \operatorname{diag}(v) \bar{Y} \bar{v}=s \\
& v_{\min , k} \leq\left|v_{k}\right| \leq v_{\max , k} \\
& p_{\min } \leq \operatorname{Re}(s) \leq p_{\max } \\
& q_{\min } \leq \operatorname{Im}(s) \leq q_{\max } \\
& \left|y_{j l}\right|\left|v_{j}-v_{l}\right| \leq i_{\max ,(j, l)} \\
& k \in \mathcal{N}, \quad(j, l) \in \mathcal{E}
\end{array}
$$

where the 1-norm cost function is proportional to the monetary cost for the power deviations introduced by the DSO. For renewable in-feed curtailment, this situation is commonplace in some European countries, where the operator is typically required by law to pay the nominal price for available power, regardless of whether it is used or curtailed. Since this is the most relevant case here, the assumption is made that all costs are of this structure. However, the general framework presented in this work can be extended to use any convex cost function. Problem (6) will hereafter be referred to as the OPF problem. It is non-convex due to the quadratic Kirchhoff equalities (6b) as well as the lower voltage magnitude bounds (6c).

\section{Difference-of-convex-functions (DC) programming}

The method used in this work for solving problem (6) is called difference-of-convex-functions $\left(\mathrm{DC}^{1}\right)$ programming. This section outlines the algorithm and presents some existing related theoretical results. DC programming is a class of algorithms for solving problems of the form

$$
\begin{aligned}
\underset{x}{\operatorname{minimize}} & g_{0}(x)-h_{0}(x) \\
\text { subject to } & g_{i}(x)-h_{i}(x) \leq 0,
\end{aligned}
$$

where $i \in\{1, \ldots, m\}$ and the $g_{i}, h_{i}$ are convex, subdifferentiable functions. A recent survey of the method and related theory is given in [17], and [15] presents the basic algorithm, which is also given in Algorithm 1 for completeness. The main idea of the algorithm is to solve a sequence of convex problems obtained by linearizing the concave parts of the constraints and objective:

$$
\begin{aligned}
\underset{x}{\operatorname{minimize}} & g_{0}(x)-\left[h_{0}(\tilde{x})+\nabla h_{0}(\tilde{x})(x-\tilde{x})\right] \\
\text { subject to } & g_{i}(x)-\left[h_{i}(\tilde{x})+\nabla h_{i}(\tilde{x})(x-\tilde{x})\right] \leq 0 .
\end{aligned}
$$

The optimizer $x^{*}$ of (8) is then used as the next point of convexification $\tilde{x}$, and the process is repeated until convergence is reached. The feasible set of (8) is a convex inner approximation of that of (7). This means that (8) is not necessarily feasible, even if the original non-convex problem is. In order to deal with this, a penalty reformulation is used:

$$
\begin{array}{cl}
\underset{x, t \geq 0}{\operatorname{minimize}} & g_{0}(x)-\left[h_{0}(\tilde{x})+\nabla h_{0}(\tilde{x})(x-\tilde{x})\right]+\beta^{k} t \\
\text { subject to } & g_{i}(x)-\left[h_{i}(\tilde{x})+\nabla h_{i}(\tilde{x})(x-\tilde{x})\right] \leq t,
\end{array}
$$

\footnotetext{
${ }^{1}$ Not to be confused with the abbreviation "DC" for direct current, and the related approximations of the AC-OPF problem.
}

1: Let $x^{0}$ initial guess, $\beta^{0}, \delta_{1}, \delta_{2}>0$ parameters, $\epsilon_{x}, \epsilon_{t}>0$ tolerances

2: while Not converged do

3: $\quad x^{k+1}, \lambda^{k+1}, t^{*} \leftarrow$ Solution of (9)

4: $\quad$ if $\left\|x^{k+1}-x^{k}\right\| \leq \epsilon_{x}$ and $t \leq \epsilon_{t}$ then

5: Terminate, converged to local optimality.

6: $\quad$ end if

7: $\quad r^{k} \leftarrow \min \left\{\left(\left\|x^{k+1}-x^{k}\right\|_{2}\right)^{-1},\left\|\lambda^{k+1}\right\|_{1}+\delta_{1}\right\}$

8: $\quad \beta^{k+1} \leftarrow \begin{cases}\beta^{k} & \text { if } \beta^{k} \geq r^{k} \\ \beta^{k}+\delta_{2} & \text { if } \beta^{k}<r^{k}\end{cases}$

9: end while

Fig. 1. Difference of convex functions algorithm from [15], modified to use practical stopping criteria. The $\lambda^{k+1}$ computed in Step 3 is the vector of dual multipliers of the constraints of the inner problem.

where $\beta^{k} \in \mathbb{R}_{+}$is a penalty weight parameter that is updated after each convexification using the rule in Algorithm 1. It is shown in [15] that this algorithm globally converges to a KKT point of (7) with a linear rate, provided one exists and standard constraint qualifications are satisfied.

\section{REFORMULATION OF OPF AS \\ DIFFERENCE-OF-CONVEX-FUNCTIONS PROBLEM}

In this section, the DSO OPF problem (6) is reformulated as a quadratically constrained linear problem (QCLP). An efficient way of computing the splits of the non-convex functions into differences of convex functions is presented, resulting in a special structure of the convex sub-problems. The latter will be efficiently solvable thanks to this structure.

\section{A. Reformulation as $Q C L P$}

As already shown in [18], OPF problems with linear cost functions can be recast as non-convex quadratically constrained linear programs. A similar technique will be applied here. First, let $s_{0} \in \mathbb{C}^{N}, v_{0} \in \mathbb{C}^{N}$ be the power and voltage vectors the system is operating at without any DSO intervention. We now introduce the difference in voltages introduced by the DSO as follows:

$$
v:=v_{0}+\Delta v \in \mathbb{C}^{N},
$$

where $\Delta v \in \mathbb{C}^{N}$ is the change from the starting point and $v$ is the resulting voltage vector. This deviation-based formulation was chosen to emphasize that the optimization is performed over changes to a set-point. Note that it would also be possible to apply the approach presented in this paper using $v$ directly. The resulting change of powers $\Delta s \in \mathbb{C}^{N}$ can be computed using the Kirchhoff equations (6b):

$$
\Delta s=\operatorname{diag}\left(v_{0}\right) \bar{Y} \bar{\Delta} v+\operatorname{diag}(\Delta v) \bar{Y} \bar{v}+\operatorname{diag}(\Delta v) \bar{Y} \bar{\Delta} v .
$$

Define now $Y^{(k)}$ as a version of $Y$ with all but the $k$-th row set to 0 . After some reformulation, we can write

$$
\begin{aligned}
& (\operatorname{Re}(\Delta s))_{k}=z^{T} H_{r, k} z+h_{r, k}^{T} z, \\
& (\operatorname{Im}(\Delta s))_{k}=z^{T} H_{q, k} z+h_{q, k}^{T} z,
\end{aligned}
$$



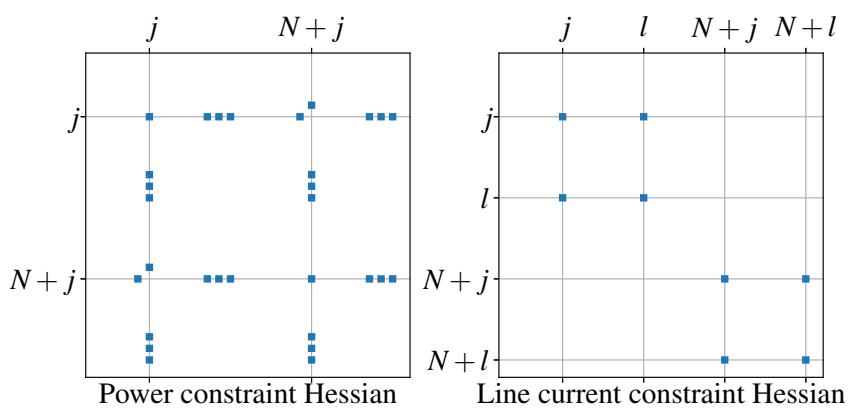

Fig. 2. Sparsity pattern of example power constraint and line constraint Hessian matrices $F$. In this case, vertex $j$ has 4 neighbors. Note that the power constraint matrix is shown in symmetric form as defined in (15).

with $z:=\left[\operatorname{Re}(\Delta v)^{T} \operatorname{Im}(\Delta v)^{T}\right]^{T} \in \mathbb{R}^{2 N}$, and

$$
\begin{aligned}
H_{r, k} & :=\left[\begin{array}{cc}
\operatorname{Re}\left(Y^{(k)}\right) & -\operatorname{Im}\left(Y^{(k)}\right) \\
\operatorname{Im}\left(Y^{(k)}\right) & \operatorname{Re}\left(Y^{(k)}\right)
\end{array}\right], \\
H_{q, k} & :=\left[\begin{array}{cc}
-\operatorname{Im}\left(Y^{(k)}\right) & -\operatorname{Re}\left(Y^{(k)}\right) \\
\operatorname{Re}\left(Y^{(k)}\right) & -\operatorname{Im}\left(Y^{(k)}\right)
\end{array}\right] .
\end{aligned}
$$

The linear parts in (11) are given by

$$
\begin{aligned}
& h_{r, k}:= \\
& {\left[\begin{array}{c}
\left(\left(v_{0}^{r}\right)_{k} g_{k}+\left(v_{0}^{q}\right)_{k} b_{k}\right)^{T}+e_{k}\left(g_{k}\left(v_{0}^{r}\right)_{k}+b_{k}\left(v_{0}^{q}\right)_{k}\right) \\
\left(\left(v_{0}^{q}\right)_{k} g_{k}-\left(v_{0}^{r}\right)_{k} b_{k}\right)^{T}+e_{k}\left(b_{k}\left(v_{0}^{r}\right)_{k}+g_{k}\left(v_{0}^{q}\right)_{k}\right)
\end{array}\right],} \\
& h_{q, k}:= \\
& {\left[\begin{array}{c}
\left(\left(v_{0}^{q}\right)_{k} g_{k}-\left(v_{0}^{r}\right)_{k} b_{k}\right)^{T}-e_{k}\left(b_{k}\left(v_{0}^{r}\right)_{k}+g_{k}\left(v_{0}^{q}\right)_{k}\right) \\
\left(-\left(v_{0}^{q}\right)_{k} b_{k}-\left(v_{0}^{r}\right)_{k} g_{k}\right)^{T}+e_{k}\left(g_{k}\left(v_{0}^{r}\right)_{k}-g_{k}\left(v_{0}^{q}\right)_{k}\right)
\end{array}\right] .}
\end{aligned}
$$

Equations (11) can now be used to express the constraints on powers given in (6d)-(6e) as constraints on $\Delta v$. Using (10), the constraints (6c) and (6f) can also be expressed in $\Delta v$. Finally, problem (6) can be rewritten entirely in the variable $z$ :

$$
\underset{z \in \mathbb{R}^{2 N}}{\operatorname{minimize}} \sum_{k=1}^{N}\left|z^{T} H_{r, k} z+h_{r, k}^{T} z\right|+\left|z^{T} H_{q, k} z+h_{q, k}^{T} z\right|
$$

$$
\text { subject to } z^{T} F_{i} z+f_{i}^{T} z+\gamma_{i} \leq 0 \text {, }
$$$$
i \in\{1, \ldots, K\} \text {, }
$$

where $K:=6 N+L$. The constraints (14b) are reformulations of the original constraints $(6 \mathrm{c})-(6 \mathrm{f})$. The structures of the $F_{i}$ are of particular importance in later sections, which is why they are given here.

(i) The matrix $F$ of power constraints (6d) and (6e) are either the matrices $H_{r, k}$ or $H_{q, k}$ or negative versions thereof.

(ii) For the voltage bounds (6c), $F$ has 1 (for upper bounds) or -1 (for lower bounds) on the $j$-th and $(N+j)$-th entries on the diagonal, and 0 everywhere else.

(iii) The matrix $F$ of line constraints (6f) have 1 in positions

$$
(j, j),(l, l),(N+j, N+j),(N+l, N+l),
$$

of the diagonal and -1 in positions

$$
(j, l),(l, j),(N+j, N+l),(N+j, N+l) .
$$

Note that the matrices from (12) are not symmetric, but they can be trivially made symmetric without changing the value of the constraints in (14b). We hence define the symmetric versions

$$
\hat{H}_{r, k}:=\frac{H_{r, k}+H_{r, k}^{T}}{2}, \quad \hat{H}_{q, k}:=\frac{H_{q, k}+H_{q, k}^{T}}{2} .
$$

A visualization of the described sparsity patterns is given in Figure 2. Since the cost function (14a) is inconvenient due to its non-smoothness, a standard 1-norm reformulation with additional slack variables $u \in \mathbb{R}^{2 N}$ is performed. Defining $x:=\left[\begin{array}{ll}z^{T} & u^{T}\end{array}\right]^{T}$, problem (14) can be written as follows:

$$
\begin{aligned}
\underset{x}{\operatorname{minimize}} & c^{T} x \\
\text { subject to } & x^{T} M_{i} x+m_{i}^{T} x+\omega_{i} \leq 0, \\
& i \in\{1, \ldots, 10 N+L\}, \\
& x_{j} \geq 0, j \in\{2 N+1, \ldots, 4 N\} .
\end{aligned}
$$

for $c, M_{i}, m_{i}, \omega_{i}$ as follows: $c$ is such that $c^{T} x=1^{T} u$. The first $6 N+L$ constraints come from (14b), hence their $\omega_{i}$ are the $\gamma_{i}$, their $m_{i}$ are the $f_{i}$ augmented with $2 N$ zero entries at the end and their $M_{i}$ are of size $4 N \times 4 N$, with the upper left block set to $F_{i}$ and the rest 0 . Similarly, the other $4 N$ constraints come from the reformulation of (14a), hence their $\omega_{i}$ are 0 , the $m_{i}$ are positive and negative versions of $h_{r, k}$ and $h_{q, k}$, augmented with $-e_{k}$ each to add the respective entry of $u$. The $M_{i}$ of the second section of constraints are positive and negative versions of $H_{r, k}, H_{q, k}$, augmented with 0 blocks as before.

\section{B. Application of DC programming}

In order to apply DC programming to solve (16), both (16a) and (16b) have to be written as a difference of two convex functions as described in (7). We call this procedure a "DC split". Since (16a) is linear, we can just define $g_{0}(x):=c^{T} x$ and $h_{0}(x):=0$. The constraints (16b) on the other hand can be non-convex, so they have to be split. For every symmetric indefinite matrix $M$, there exist infinitely many pairs $M^{+}, M^{-} \succeq 0$ such that

$$
M=M^{+}-M^{-} .
$$

This means that once such $M^{+}$and $M^{-}$are determined for all constraints, problem (16) can now be written in the form of (7) and the algorithm from Figure 1 can directly be applied. The existence of infinitely many splits of the $M$ matrices from (16) raises the question of optimal split selection. The selection that has been effective in experiments is to split the matrices such that the $M_{i}^{-}$have small eigenvalues. This split strategy leads to the curvature of the concave terms $-x^{T} M_{i}^{-} x$ being smaller and therefore the linearized approximation being closer to the original nonconvex term. 


\section{Analytic eigenvalue computations}

Since there are a large number of constraints of the type (16b), calculating splits using numerical eigenvalue decompositions would be computationally prohibitive. Due to the structure of the $M_{i}$, eigenvalues can be computed analytically using the method described in this section. Note first that for the indexes $i$ corresponding to voltage or line constraints, the eigenvalues of $M_{i}$ are trivial to compute due to their simple structure. For the power constraints, we use the following Lemma (previously shown in [19]):

Lemma 1 The eigenvalues of the matrices $\hat{H}_{r, k}$ from (15) are given by

$$
\begin{aligned}
& \operatorname{eig}\left(\hat{H}_{r, k}\right)=\left\{\frac{\operatorname{Re}\left(Y_{k k}\right) \pm \sqrt{\operatorname{Re}\left(Y_{k k}\right)^{2}-4\left\|Y^{(k)}\right\|_{2}^{2}}}{2}, 0\right\}, \\
& \operatorname{eig}\left(\hat{H}_{q, k}\right)=\left\{\frac{-\operatorname{Im}\left(Y_{k k}\right) \pm \sqrt{\operatorname{Im}\left(Y_{k k}\right)^{2}-4\left\|Y^{(k)}\right\|_{2}^{2}}}{2}, 0\right\} .
\end{aligned}
$$

Proof: See [19], Section 5.3.

Note that the computation of the eigenvalues above is particularly efficient since the $Y^{(k)}$ only have as many entries as bus $k$ has neighbors. Since power system graphs are generally very sparse, this yields a significant reduction in computational cost over even an iterative approximation of eigenvalues, let alone a standard exact computation. This decomposition has to be performed only once for a given network topology. The computational effort is negligible compared to the rest of the algorithm.

\section{Sparse splits}

At this point, the eigenvalues of all the matrices $M_{i}$ can be computed efficiently. In this section, these eigenvalues will be used to construct $M_{i}^{+}$and $M_{i}^{-}$efficiently. We define a sparse, diagonal matrix $D_{i}$ as follows:

$$
\left(D_{i}\right)_{j j}= \begin{cases}1, & \text { if } j \in J\left(M_{i}\right) \cup J\left(m_{i}\right), \\ 0, & \text { otherwise. }\end{cases}
$$

This diagonal matrix $D_{i}$ hence has ones only at the row and column indexes at which at least one of $M_{i}$ or $m_{i}$ also has nonzeros. We then define the split

$$
M_{i}:=\underbrace{\alpha D_{i}}_{M_{i}^{+}}-\underbrace{\left(\alpha D_{i}-M_{i}\right)}_{M_{i}^{-}},
$$

where $\alpha$ is the absolute value of the largest eigenvalue of $M_{i}$. This sparse split guarantees positive definiteness of the split matrices since it only shifts the non-zero eigenvalues. Experiments showed that this splitting choice both leads to sparse, well-structured inner problems as well as leads to a reasonable number of outer iterations. Adding a regularization $\epsilon I, \epsilon>0$ to both $M^{+}$and $M^{-}$leads to the inner problems becoming numerically easier, but leads to a loss of sparsity of the latter. Other split choices were not explored.

\section{EFFICIENT SOLUTION OF INNER PROBLEMS}

The bottleneck of the DC algorithm is the solution of the convex approximation. With the splits (21) applied, the problem to be solved at each DC iteration has the form

$$
\begin{aligned}
\underset{x \in \mathbb{R}^{4 N}, t \in \mathbb{R}}{\operatorname{minimize}} & c^{T} x+\beta^{k} t \\
\text { subject to } & x^{T} M_{i}^{+} x+\hat{m}_{i}\left(\tilde{x}^{k}\right)^{T} x+\hat{\omega}_{i}\left(\tilde{x}^{k}\right) \leq t, \\
& i \in\{1, \ldots, 10 N+L\}, \quad t \geq 0, \\
& x_{j} \geq 0, \quad j \in\{2 N+1, \ldots, 4 N\},
\end{aligned}
$$

where $\tilde{x}^{k}$ is the current point around which a convex approximation is formed, and

$$
\begin{aligned}
\hat{m}_{i}\left(\tilde{x}^{k}\right) & :=\left(m_{i}-2 M_{i}^{-} \tilde{x}^{k}\right), \\
\hat{\omega}_{i}\left(\tilde{x}^{k}\right) & :=\omega_{i}+\left(\tilde{x}^{k}\right)^{T} M_{i}^{-} \tilde{x}^{k} .
\end{aligned}
$$

General-purpose sparse convex second-order cone programming codes such as ECOS [20], GUROBI [21] or MOSEK [22] can be used to solve these problems. However, the structure of the problem suggests that a specialized solver could lead to increased performance: The constraint Hessians $M_{i}^{+}$are diagonal and sparse, and all nonzero entries have the same values. Exploiting this structure and using some additional reformulation, the dual of (22) can be written as

$$
\begin{array}{ll}
\underset{\lambda}{\operatorname{minimize}} & \frac{1}{4} \lambda^{T} C\left(\operatorname{diag}\left(D^{T} \lambda\right)^{-1}\right) C^{T} \lambda+d^{T} \lambda \\
\text { subject to } & 0 \leq \lambda \leq 1 .
\end{array}
$$

A detailed derivation of the problem data in (24) is given in the appendix. Problem (24) can be solved efficiently using the projected gradient method [23], [24]. Due to the sparsity of $C$ and $D$, the iteration complexity of the method is linear in the number of buses and lines of the grid if the maximum number of neighbors in the grid is assumed to grow slowly with grid size.

\section{NumericAl RESUlts}

In this section, we present numerical results on the performance and behavior of the proposed algorithm. In order to make the results comparable to other work in the field, some of the tests will be conducted on the IEEE benchmark test systems available in MATPOWER [6], [25]. For the experiments, a standalone implementation of the proposed method was created, which will be referred to as DQ-OPF. The implementation is a single-threaded, library-free ANSI $\mathrm{C}$ code. The test computer had a Core i7-4600U dual-core CPU clocked at $2.1 \mathrm{GHz}$ and $8 \mathrm{~GB}$ of memory. The operating system used was Debian Linux.

\section{A. Algorithm behavior}

In the first set of results, the convergence behavior of the algorithm is investigated. For these problems, a local optimum $\left(v^{*}, s^{*}\right)$ was found with IPOPT [26]. The entries of $v^{*}$ were then perturbed uniformly and the corresponding perturbed powers were computed using the Kirchhoff equations to yield a perturbed operating point $(\tilde{v}, \tilde{s})$. The perturbation size was chosen to make the maximum power 

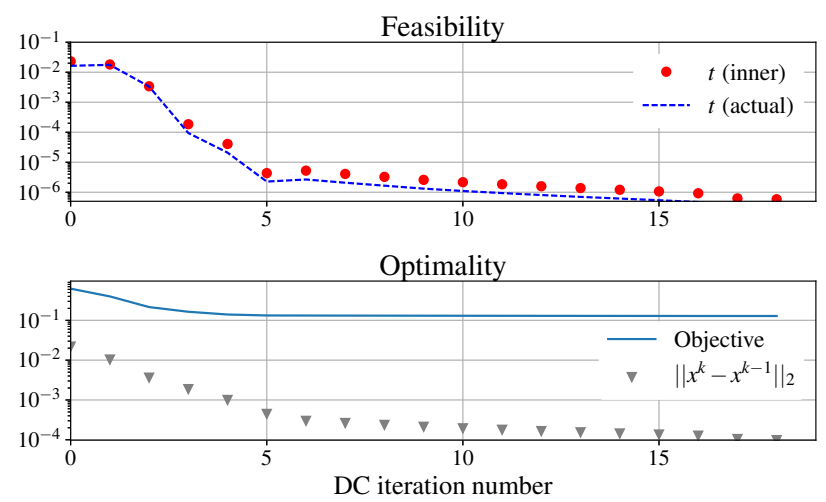

Fig. 3. The DC method applied to the MATPOWER version of the IEEE 30-bus grid, using dual projected gradient as the inner solver. The " $t$ (inner)" line represents the maximum constraint violation of the convex approximation at that iteration, whereas the " $t$ (actual)" represents the maximum constraint violation of the original, non-convex problem. The lower subplot shows the true objective as well as the difference between subsequent iterates.
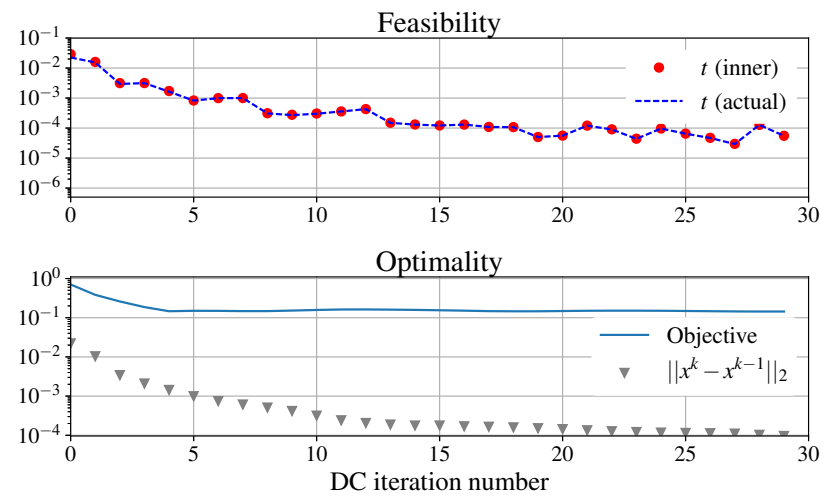

Fig. 4. Solution of the same problem as in Figure 3, but with the accuracy for the inner problem severely limited. The optimal value of the inner problems no longer decreases monotonically, due to the inaccurate inner solutions.

bound violation about $100 \%$. This was done in order to simulate the practical situation of the power grid state being only slightly infeasible with respect to the operational constraints, but respecting the Kirchhoff equations. Note that a point satisfying the Kirchhoff equations can be found for any initial guess of voltages by simple evaluation of equation (2). While satisfaction of the Kirchoff equations is not a prerequisite for the method to work, the former was found to improve convergence speed. The point $(\tilde{s}, \tilde{v})$ was used as starting point $\left(s_{0}, v_{0}\right)$ as defined in Section II-B, and DQ-OPF started from there.

An example solver run is shown in Figure 3 with a termination criterion of $\left\|x^{k}-x^{k-1}\right\|_{2} \leq 10^{-4}$. Within a small number of DC iterations, the maximum constraint violation of the non-convex problem drops below $10^{-4}$ per unit, which is well below $1 \%$ relative accuracy. Note also that the objective value does not improve significantly past iteration 5. For the problem shown in Figure 3, the inner
TABLE I

AVERAGE TIME AND OBJeCTIVE FOR $1 \%$ RELATIVE ACCURACY

\begin{tabular}{lcc|cc}
\hline & \multicolumn{2}{c}{ IPOPT \& PARDISO } & \multicolumn{2}{c}{ DQ-OPF } \\
& Time & Objective & Time & Objective \\
6-bus & $55 \mathrm{~ms}$ & 0.9 & $2.2 \mathrm{~ms}$ & 0.9 \\
9-bus & $65 \mathrm{~ms}$ & 1.9 & $2.3 \mathrm{~ms}$ & 1.5 \\
14-bus & $68 \mathrm{~ms}$ & 1.3 & $3.5 \mathrm{~ms}$ & 1.0 \\
30-bus & $77 \mathrm{~ms}$ & 1.5 & $10 \mathrm{~ms}$ & 1.3 \\
39-bus & $92 \mathrm{~ms}$ & 12 & $23 \mathrm{~ms}$ & 11 \\
57-bus & $97 \mathrm{~ms}$ & 2.9 & $25 \mathrm{~ms}$ & 1.6 \\
118-bus & $213 \mathrm{~ms}$ & 17 & $76 \mathrm{~ms}$ & 3.7 \\
2383-bus & $3.5 \mathrm{~s}$ & 24 & $4.4 \mathrm{~s}$ & 10.4 \\
2737-bus & $3.3 \mathrm{~s}$ & 12 & $2.4 \mathrm{~s}$ & 7.6 \\
3210-bus & $2.8 \mathrm{~s}$ & 14 & $4.0 \mathrm{~s}$ & 9.2 \\
9241-bus & $15 \mathrm{~s}$ & 26 & $16 \mathrm{~s}$ & 6.8 \\
\hline
\end{tabular}

convex problems were solved to high accuracy $\left(10^{4}\right.$ inner iterations). In other sequential convex programming methods, it is often observed that solving the intermediate problems approximately can often be sufficient for convergence [27]. In order to investigate if the proposed method behaves similarly, the gradient solver iterations were limited to 100 in the same problem as above, and the other parameters left unchanged. The resulting run for the same problem is shown in Figure 4. While the number of outer iterations required is higher than before for the same accuracy, the overall computations are two orders of magnitude cheaper computationally. There is a trade-off between inner accuracy and outer number of iterations: Limiting the inner accuracy too much can lead to a strongly increased amount of outer iterations, especially for larger grids. It was found that the inner accuracy should be chosen at least as accurate as the termination criteria of the outer loop. Note that the objectives in Figure 3 and Figure 4 converge to slightly different values. This is due to the two solver runs converging to different local optima. In the experiments with the proposed method, it was found that the KKT conditions were satisfied whenever the method was run until convergence, however in general the second-order condition indicated that convergence was to saddle points and occasionally local minima. Convergence to local maxima was never observed.

The specific KKT point converged to depends on the starting point of the method as well as the algorithm parameters. Additionally, if the method is started at a point that is very far away from being feasible (for example, all voltages set to 0 ), it can converge to a locally infeasible point. Both of these properties are shared by all other local methods for solving non-convex problems the authors are aware of. The latter case of convergence to local infeasibility was only observed for the largest systems in experiments with the aforementioned bad starting points.

\section{B. Performance}

In order to compare the implemented method to the state of the art, MATPOWER test cases were used in conjunction with the 1-norm cost function, as described in (6). The 1- 
norm cost was implemented using MATPOWER's piecewise affine cost function functionality. Instead of solving the inner problems accurately as shown in Figure 3, the inner solver was limited to 100-1000 iterations depending on grid size, yielding the aforementioned calculation time improvements. The DC solver parameters were tuned for one instance of the problem and then reused across all runs. The solver was started at $\left(s_{0}, v_{0}\right)$. As a reference, we used MATPOWER's IPOPT interface along with the parallel PARDISO [28]-[30] solver for linear systems. This reference solver was selected because Matpower's website listed it as the best performing solver in the package. Table I presents the results averaged over 100 runs with random initial points created as in the previous experiment. In these experiments, IPOPT was warmstarted at the same point as DQ-OPF using MATPOWERs warm-start functionality. DQ-OPF is faster in many cases, with the speedups for the smaller grids being substantial. For the larger grids, the run times are comparable to the reference. Further speedups can be expected in the proposed method through parallelization and more efficient code. For the largest grid, MATPOWER ran into memory issues on the computer used. DQ-OPF uses a memory amount linear in the problem size and hence had no such issues. Note also that IPOPT was run with multi-threading enabled ( 2 threads) and the times shown are wall clock, not CPU time.

Another observation is that the average objective values were consistently smaller with the method used. This means the proposed method found local optima with better objective values. A likely reason for this is the objective function, which represents distance from the starting point $\left(s_{0}, v_{0}\right)$. The presented method tends to find local optima close to the point at which it was started, whereas IPOPT (and interiorpoint methods in general) seem to benefit less from warmstart information [31]. This difference in objective values was made both when IPOPT was warm-started as well as when the default settings (no warm-start) were used.

Further experiments showed that the runtime of the proposed method increases roughly linearly in the distance of the starting point to the KKT point it converges to. For the larger systems, the increase in runtime was found to be larger than for the smaller systems. It may therefore be advisable to use an alternative method in cases where no good initial guess for the voltage profile is available. Note that in time simulations an initial guess is always available in the form of the solution of the previous time step.

\section{Case study: Simulation experiment}

In order to demonstrate the effectiveness of warm-starting the presented method, a power system time simulation experiment is presented in this section. The experiment was run with the test grid shown in Figure 7. Three different approaches to dealing with voltage violations were tested:

(i) Rule-based curtailment: In this control scheme, no optimization is run, instead the renewable in-feeds of the grid are simply curtailed down to a fixed fraction of their rated in-feed. This case reflects current industry practice.
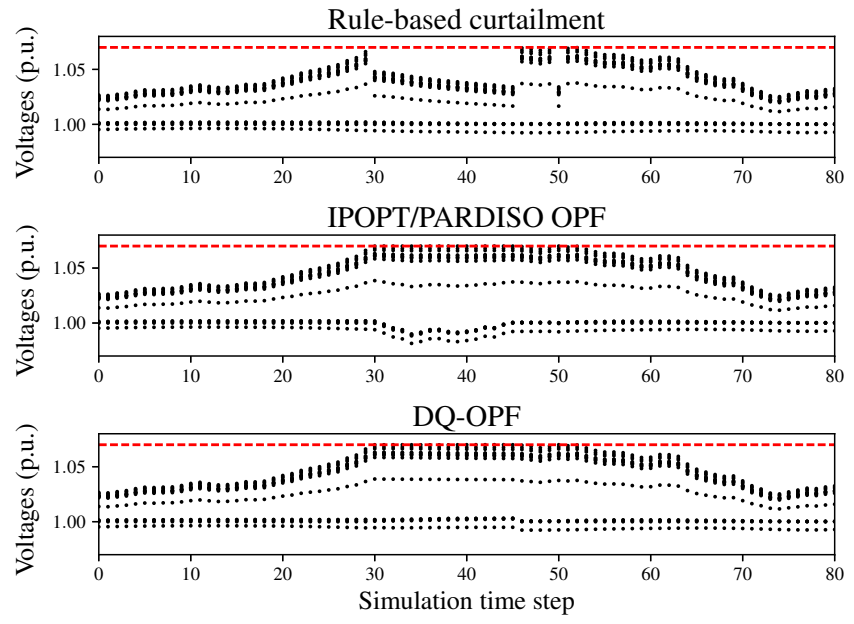

Fig. 5. Voltage traces for all buses in the MV grid over the course of the simulation. Admissible limits were $[0.9,1.07]$ per unit. The OPF problem had to be solved at time instances 30 through 45 and 50 .

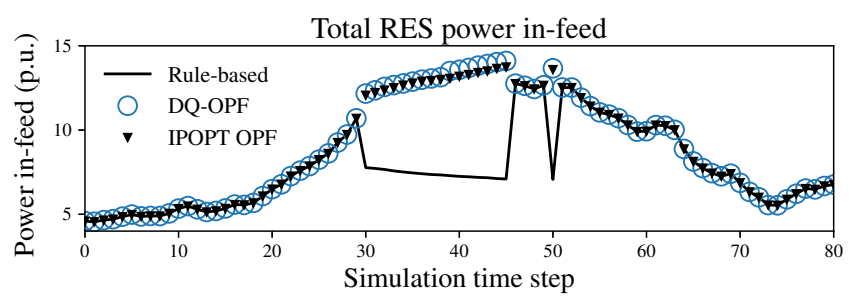

Fig. 6. Total RES power in-feed for the MV grid over the simulation horizon. The MATPOWER and proposed OPF solutions are close, with the proposed solution discarding slightly less renewable energy.

(ii) IPOPT OPF-based curtailment: In this scheme, problem (6) is solved to local optimality with MATPOWER, using IPOPT/PARDISO as OPF solver.

(iii) Proposed method OPF-based curtailment: Problem (6) is solved to local optimality, but with the method presented in this paper. The solver is warm-started with the solution from the previous solve when available. The inner problems were solved with the dual gradient method, which was limited to 200 inner iterations.

As a simulation environment, Adaptricity DPG.sim [34] was used. At each simulation time step, the operational limits (2), (3) and (4) were checked. If any of them were violated, one of the approaches above was invoked. The resulting power in-feed and voltage profiles for the different approaches are shown in Figures 5 and 6, respectively. As can be seen in the uppermost subplots of the two figures, the profiles obtained by using the rule-based curtailment controller have strong fluctuations due to the controller intervening non-smoothly when violations are detected. Both the voltage and power profiles are much smoother if the optimizationbased intervention solving (6) is performed. Even though only local optima are found both in MATPOWER and the presented method, these smoother profiles were observed in all simulations. Additionally, even though the different numerical approaches often yield different local minima, the 


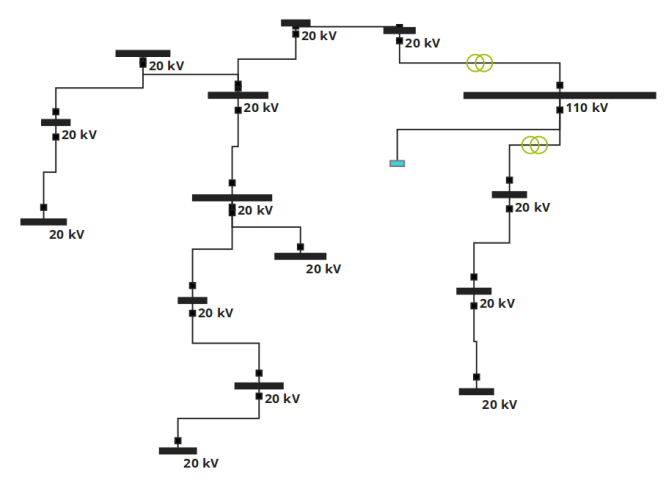

Fig. 7. Rural MV grid used for the simulation experiment. Taken from [32], with cycles opened. The system data is available at [33]. Note that while the grid here is radial, this is not a required assumption for the proposed method. The cyan bus in the middle is the slack bus, modeled here as a bus with no power limits.

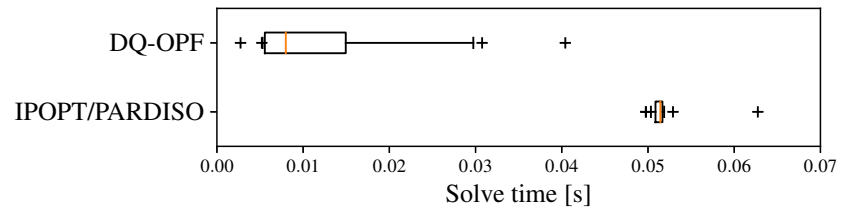

Fig. 8. Box-plot of the distribution of solve times for the optimization problems solved in the simulation experiment. The boxes contain $50 \%$ of the cases, the interval marked by the horizontal lines contains $90 \%$ of the cases. The plus signs mark outliers.

difference in cost function values is minor. The distribution of solve times for the simulation is presented in Figure 8. As can be seen, the average solve time of the proposed method is only about $14 \%$ of the state of the art. This directly results in a speedup of up to factor 7 in the simulations.

Finally, due to the less severe interventions, much less curtailment is required compared to the rule-based controller, resulting in a significant increase of renewable energy integrated. The typical increase in the latter is in the $20-40 \%$ in yearly simulations, but the specific value depends strongly on the grid topology and available amount of RES capacity.

\section{CONCLUSION}

This paper presented an alternative approach to dealing with over-voltage problems in distribution grids with an OPF-based approach that leads to minimum intervention by the DSO. Along with a formulation of the optimization problem, a novel method to solve it to local optimality was presented. The latter can be warm-started and significantly outperforms current state-of-the-art interior-point methods for small size test systems. The presented method can easily be extended to other optimization problems involving $\mathrm{AC}$ power flow constraints.

\section{ACKNOWLEDGMENTS}

This work was supported by the Swiss Commission for Technology and Innovation (CTI), (Grant 16946.1 PFIWIW). We thank the team at Adaptricity (Stephan Koch, An- dreas Ulbig, Francesco Ferrucci) for providing the simulation environment and valuable discussions on power systems.

\section{APPENDIX}

\section{A. Box-constrained inner problem formulation}

The intersection of the constraints in (22) is not efficient to project onto, hence using the projected gradient method will not be efficient. Using two reformulations, the problem will be recast as a minimization of a smooth function subject to box constraints. The first step is a lifting into a higherdimensional variable space: We introduce variables $y_{i}:=x_{i}^{2}$ and change the penalty function from an $\infty$-norm to a 1 norm (another possible penalty function shown in [15]). The inner problem to be solved then becomes

$$
\begin{array}{cl}
\underset{x, y, t}{\operatorname{minimize}} & c^{T} x+\beta^{k} 1^{T} t \\
\text { subject to } & A x+B y-b \leq t, \\
& \operatorname{diag}(x) x-y=0, \\
& t \geq 0, x_{j} \geq 0, \\
& j \in\{2 N+1, \ldots, 4 N\},
\end{array}
$$

where

$$
A:=\left[\begin{array}{c}
\hat{m}_{1}\left(\tilde{x}^{k}\right)^{T} \\
m_{2}\left(\tilde{x}^{k}\right)^{T} \\
\vdots \\
m_{K}\left(\tilde{x}^{k}\right)^{T}
\end{array}\right], B:=\left[\begin{array}{c}
\operatorname{diag}\left(M_{1}^{+}\right)^{T} \\
\operatorname{diag}\left(M_{2}^{+}\right)^{T} \\
\vdots \\
\operatorname{diag}\left(M_{K}^{+}\right)^{T}
\end{array}\right],
$$

and $b:=\left[\begin{array}{llll}-\hat{\omega}_{1}\left(\tilde{x}^{k}\right) & -\omega_{2}\left(\tilde{x}^{k}\right) & \ldots & -\omega_{K}\left(\tilde{x}^{k}\right)\end{array}\right]^{T}$, where $K$ is $10 N+L$. Note that since $u_{i}^{2}$ does not appear anywhere, the last $2 N$ columns of $B$ are 0 . We now make use of the following lemma to relax the constraints in $(25 \mathrm{c})$ :

Lemma 2 Consider a version $(P)$ of (25) with the constraints $(25 \mathrm{c})$ relaxed to

$$
\operatorname{diag}(x) x-y \leq 0 .
$$

For every minimizer of $(P)$ with one or more of (27) inactive, a minimizer with equal cost function can be found which has all the constraints (27) active.

Proof: The lemma will be shown by construction: Assume a point $\left(x^{\star}, y^{\star}, t^{\star}\right)$ is optimal for $(\mathrm{P})$, but a constraint in (27) is not active. Then the $y_{i}$ corresponding to that constraint can be decreased to make the constraint active without any change to the cost function or constraint satisfaction. The latter is due to all entries of $B$ being non-negative.

Lemma 2 implies that we can simply solve the relaxed version of (25) and then recover an optimal solution for the latter. This is not surprising, since (25) is just a reformulation of a convex problem.

\section{B. Derivation of the dual problem}

In this section, a detailed derivation of the step from (25) to (24) is given. First, notice that the only non-zeros entries of $c$ in (25) are those corresponding to the slack variables $u$ introduced in (16). Moreover, the constraints corresponding to the cost function reformulation do not need penalties, 
since they are satisfied by construction. Recalling $x=$ $\left[\begin{array}{ll}z^{T} & u^{T}\end{array}\right]^{T}$, we can rewrite (25) as

$$
\begin{array}{cl}
\underset{x, \tilde{y}, u, t}{\operatorname{minimize}} & 1^{T} u+\beta^{k} 1^{T} t \\
\text { subject to } & A_{1} z+B_{1} \tilde{y}-b_{1} \leq t, \\
& A_{2} z+B_{2} \tilde{y}-b_{2} \leq u, \\
& \operatorname{diag}(z) z-\tilde{y} \leq 0, \\
& t \geq 0, u \geq 0,
\end{array}
$$

where $t$ is now a vector and $\tilde{y}$ is a vector of the first $2 N$ entries of $y$ (the last $2 N$ entries would represent $u_{i}^{2}$ and can be removed from the problem). In (28), constraints (28b) contain all the actual constraints (originally (14b)), whereas (28c) contains all constraints resulting from the cost function reformulation. The matrices $A_{1}, A_{2}$ are the rows of the respective constraints in (25b), with the last $2 N$ columns removed because $u$ is now treated separately again. In the 1-norm cost formulation, a trick has been applied: Normally, a cost of $|w|$ for some variable $w$ would be replaced by one slack variable $s$ and then a problem

$$
\begin{gathered}
\underset{w, s}{\operatorname{minimize}} \quad s+(\text { other costs) } \\
\text { subject to } w \leq s,-w \leq s,
\end{gathered}
$$

(other constraints),

solved. An equivalent formulation to this is to introduce two slack variables $s_{1}, s_{2}$ and solve

$$
\begin{aligned}
\underset{w, s}{\operatorname{minimize}} & s_{1}+s_{2}+\text { (other costs) } \\
\text { subject to } & w \leq s_{1},-w \leq s_{2} \\
& s_{1} \geq 0, s_{2} \geq 0
\end{aligned}
$$

(other constraints).

The equivalence is easily shown: One of $-w, w$ is always negative, leading to one of $s_{1}, s_{2}$ becoming 0 , and the cost being equivalent to the more standard formulation. Because this alternative formulation was used, one can treat the $t$ and $u$ in (28) the same and rewrite the latter once more as

$$
\begin{aligned}
\underset{x, \tilde{y}, t}{\operatorname{minimize}} & 1^{T} t \\
\text { subject to } & C z+D \tilde{y}-d \leq t, \\
& \operatorname{diag}(z) z-\tilde{y} \leq 0, \\
& t \geq 0,
\end{aligned}
$$

where $t \in \mathbb{R}^{10 N+L}$ and

$$
C:=\left[\begin{array}{c}
\beta^{k} A_{1} \\
A_{2}
\end{array}\right], \quad D:=\left[\begin{array}{c}
\beta^{k} B_{1} \\
B_{2}
\end{array}\right], \quad d:=\left[\begin{array}{c}
\beta^{k} b_{1} \\
b_{2}
\end{array}\right] .
$$

At this point, let $\lambda, \mu$ and $\gamma$ be the dual multipliers for the constraints (29b), (29c) and (29d), respectively. The Lagrangian of (29) then becomes

$$
\begin{aligned}
L(z, \tilde{y}, t, \lambda, \mu, \gamma)= & 1^{T} t+\lambda^{T}(C z+D \tilde{y}-d-t) \\
& +\mu^{T}(\operatorname{diag}(z) z-\tilde{y})+\gamma(-t) .
\end{aligned}
$$

Setting the partial derivatives to 0 yields

$$
\begin{aligned}
1-\lambda-\gamma & =0, \\
C^{T} \lambda+2 \operatorname{diag}(\mu) z & =0, \\
D^{T} \lambda-\mu & =0 .
\end{aligned}
$$

Equation (31b) implies that

$$
z^{*}(\lambda, \mu)=-\frac{1}{2} \operatorname{diag}(\mu)^{-1} C^{T} \lambda .
$$

This relation can also be used to recover the primal variables from a dual solution. The dual problem hence becomes

$$
\begin{aligned}
\underset{x, y, t}{\operatorname{maximize}} & -\frac{1}{4} \lambda^{T} C \operatorname{diag}(\mu)^{-1} C^{T} \lambda-d^{T} \lambda \\
\text { subject to } & \lambda, \gamma, \mu \geq 0, \\
& 1-\lambda-\gamma=0, \\
& D^{T} \lambda-\mu=0 .
\end{aligned}
$$

Upon closer inspection of (32), it can be seen that $\gamma$ and $\mu$ can be eliminated to yield

$$
\begin{aligned}
\underset{x, y, t}{\operatorname{maximize}} & -\frac{1}{4} \lambda^{T} C \operatorname{diag}\left(D^{T} \lambda\right)^{-1} C^{T} \lambda-d^{T} \lambda \\
\text { subject to } & 0 \leq \lambda \leq 1, \\
& D^{T} \lambda \geq 0 .
\end{aligned}
$$

Finally, since all entries of $D$ are non-negative, constraints (33b) imply (33c), and the latter can therefore be removed, resulting in the formulation (24) presented in the main text.

\section{REFERENCES}

[1] REN21, "Renewables 2015: Global status report," [Online]. Available: http://www.ren21.net/, 2015.

[2] H. Ayres, W. Freitas, M. D. Almeida, and L. D. Silva, "Method for determining the maximum allowable penetration level of distributed generation without steady-state voltage violations," IET Generation, Transmission \& Distribution, vol. 4, pp. 495-508(13), April 2010.

[3] J. Warrington, C. Hohl, P. Goulart, and M. Morari, "Optimal unit commitment accounting for robust affine reserve policies," in American Control Conference (ACC), 2014, (Portland, OR, USA), pp. 50495055, June 2014.

[4] A. Ulbig and G. Andersson, "On operational flexibility in power systems," in Power and Energy Society General Meeting, (San Diego, CA, USA), pp. 1-8, IEEE, 2012.

[5] E. Vrettos, F. Oldewurtel, M. Vasirani, and G. Andersson, "Centralized and decentralized balance group optimization in electricity markets with demand response," in PowerTech, (Grenoble, France), pp. 1-6, IEEE, 2013.

[6] R. Zimmerman, C. Murillo-Sánchez, and R. Thomas, "MATPOWER: Steady-State Operations, Planning, and Analysis Tools for Power Systems Research and Education," IEEE Transactions on Power Systems, vol. 26, pp. 12-19, Feb 2011.

[7] S. Frank, I. Steponavice, and S. Rebennack, "Optimal power flow: A bibliographic survey I," Energy Systems, vol. 3, no. 3, pp. 221-258, 2012.

[8] S. Frank, I. Steponavice, and S. Rebennack, "Optimal power flow: A bibliographic survey II," Energy Systems, vol. 3, no. 3, pp. 259-289, 2012.

[9] G. Torres and V. Quintana, "On a nonlinear multiple-centralitycorrections interior-point method for optimal power flow," IEEE Transactions on Power Systems, vol. 16, pp. 222-228, May 2001.

[10] O. Alsac, J. Bright, M. Prais, and B. Stott, "Further developments in LP-based optimal power flow," IEEE Transactions on Power Systems, vol. 5, pp. 697-711, Aug 1990. 
[11] S.-K. Chang, F. Albuyeh, M. L. Gilles, G. E. Marks, and K. Kato, "Optimal real-time voltage control," IEEE Transactions on Power Systems, vol. 5, no. 3, pp. 750-758, 1990.

[12] S. Robinson, "A quadratically-convergent algorithm for general nonlinear programming problems," Mathematical Programming, vol. 3, no. 1 , pp. $145-156,1972$

[13] J. Lavaei and S. Low, "Zero duality gap in optimal power flow problem," IEEE Transactions on Power Systems, vol. 27, pp. 92-107, Feb 2012.

[14] D. Molzahn, J. Holzer, B. Lesieutre, and C. DeMarco, "Implementation of a large-scale optimal power flow solver based on semidefinite programming," IEEE Transactions on Power Systems, vol. 28, pp. 3987-3998, Nov 2013.

[15] H. Le Thi, V. Huynh, and T. Dinh, "DC Programming and DCA for General DC Programs," in Advanced Computational Methods for Knowledge Engineering, vol. 282 of Advances in Intelligent Systems and Computing, pp. 15-35, Springer International Publishing, 2014.

[16] BMWI, "Gesetz für den Ausbau erneuerbarer Energien (EEG)," [Online]. Available: http://www.bmwi.de, 2014

[17] L. An and P. Tao, "The DC (Difference of Convex Functions) Programming and DCA Revisited with DC Models of Real World Nonconvex Optimization Problems," Annals of Operations Research, vol. 133, no. 1-4, pp. 23-46, 2005.

[18] S. H. Low, "Convex Relaxation of Optimal Power Flow, Part I: Formulations and Equivalence," arXiv preprint arXiv:1405.0766, 2014.

[19] J. D. Foster, Mixed-integer quadratically-constrained programming, piecewise-linear approximation and error analysis with applications in power flow. PhD thesis, The University of Newcastle, Australia, School of Mathemastical and Physical Sciences, 2013

[20] A. Domahidi, E. Chu, and S. Boyd, "ECOS: An SOCP solver for embedded systems," in European Control Conference (ECC), (Zurich, Switzerland), pp. 3071-3076, 2013.

[21] I. Gurobi Optimization, Gurobi Optimizer Reference Manual, 2014.

[22] ApS, The MOSEK C optimizer API manual Version 7.0, 2015.

[23] Y. Nesterov, "A method of solving a convex programming problem with convergence rate o (1/k2)," Soviet Mathematics Doklady, vol. 27, no. 2, pp. 372-376, 1983.

[24] S. Richter, Computational Complexity Certification of Gradient Methods for Real-Time Model Predictive Control. PhD thesis, ETH Zurich, 2012.

[25] S. Fliscounakis, P. Panciatici, F. Capitanescu, and L. Wehenkel, "Contingency ranking with respect to overloads in very large power systems taking into account uncertainty, preventive, and corrective actions," Power Systems, IEEE Transactions on, vol. 28, no. 4, pp. 4909-4917, 2013.

[26] A. Wächter and L. T. Biegler, "On the implementation of an interiorpoint filter line-search algorithm for large-scale nonlinear programming," Mathematical programming, vol. 106, no. 1, pp. 25-57, 2006.

[27] M. Heinkenschloss and L. N. Vicente, "Analysis of inexact trustregion SQP algorithms," SIAM Journal on Optimization, vol. 12, no. 2, pp. 283-302, 2002.

[28] A. Kuzmin, M. Luisier, and O. Schenk, "Fast methods for computing selected elements of the greens function in massively parallel nanoelectronic device simulations," in Euro-Par 2013 Parallel Processing (F. Wolf, B. Mohr, and D. Mey, eds.), vol. 8097 of Lecture Notes in Computer Science, pp. 533-544, Springer Berlin Heidelberg, 2013.

[29] O. Schenk, M. Bollhöfer, and R. A. Römer, "On Large-Scale Diagonalization Techniques for the Anderson Model of Localization," SIAM Rev., vol. 50, pp. 91-112, Feb. 2008.

[30] O. Schenk, A. Wächter, and M. Hagemann, "Matching-based preprocessing algorithms to the solution of saddle-point problems in large-scale nonconvex interior-point optimization," Computational Optimization and Applications, vol. 36, no. 2-3, pp. 321-341, 2007.

[31] E. John and E. Yıldırım, "Implementation of warm-start strategies in interior point methods for linear programming in fixed dimension," Computational Optimization and Applications, vol. 41, no. 2, pp. 151183,2008

[32] K. Strunz, S. Barsali, and Z. Styczynski, "Cigre task force c6. 04.02: Developing benchmark models for integrating distributed energy resources," in Proceedings of the CIGRE 5th Southern Africa regional conference: study committee C6 colloquium, 2005.

[33] S. Merkli, "Benchmark system data," [Online]. Available: http://control.ee.ethz.ch/\%7Esmerkli/case15bench.m doi:10.3929/ethzb-000533853, 2016 [updated: 23.02.2022].
[34] S. Koch, F. Ferrucci, A. Ulbig, and M. Koller, "Time-series simulations and assessment of smart grid planning options of distribution grids," in Proceedings of the CIRED Workshop, (Rome, Italy), 2014. 